http://jmscr.igmpublication.org/home/

ISSN (e)-2347-176x ISSN (p) 2455-0450

crossref DOI: https://dx.doi.org/10.18535/jmscr/v8i7.77

Journal Of Medical Science And Clinical Research

IGM Publication

An official Publication of IGM Publication

\title{
Interrelationship among High Sensitivity C-Reactive Protein (Hs-CRP), Glycosylated Hemoglobin (HbA1C), Gamma Glutamyl Transferase (GGT) and Microvascular Complications in Type 2 Diabetes Mellitus
}

\author{
Authors \\ Shivani Sharma ${ }^{*}$, Himani Sharma ${ }^{2}$ \\ ${ }^{1}$ Vardhman Mahavir Medical College \& Safdarjung Hospital, New Delhi, India \\ ${ }^{2}$ Government Medical College, Amritsar, Punjab, India \\ *Corresponding Author \\ Shivani Sharma
}

\begin{abstract}
Background: Diabetes Mellitus, a hyperglycemic state, leads to inflammatory reaction and oxidant injury with progression to vascular complications. High sensitivity C-Reactive protein (Hs-CRP), a marker of inflammation; gamma glutamyl transferase (GGT), a marker of oxidant injury; glycosylated hemoglobin (HbAlC), a marker of glycemic control and urine albuminuria (qualitative) as an assessment of diabetic microvascular complications can be easily studied.

Aim: To study interrelationship among $\mathrm{Hs}$-CRP, HbAlC, GGT and microvascular complications in type 2 diabetes mellitus patients and to establish role of GGT and Hs-CRP as early markers for development of diabetic microvascular complications. These markers together have not been studied in past.

Method: It was a hospital based cross-sectional case-control study conducted in tertiary care hospital on 75 type 2 diabetic patients selected by inclusion and exclusion criteria with 50 age and sex matched healthy controls. Quantitative assessment of $\mathrm{Hs}-\mathrm{CRP}, \mathrm{GGT}, \mathrm{HbAlC}$ and urine albuminuria(qualitative) was done.

Results: Mean value of $\mathrm{Hs}-\mathrm{CRP}, \mathrm{GGT}$ and $\mathrm{HbAlC}$ in diabetic cases was $8.94 \pm 2.54 \mathrm{mg} / \mathrm{L}, 101.61 \pm$ $36.84 \mathrm{U} / \mathrm{L}, 9.24 \pm 1.97 \%$ respectively in comparison to controls $(1.52 \pm 0.98 \mathrm{mg} / \mathrm{L}, 19.06 \pm 8.77 \mathrm{U} / \mathrm{L}$, $4.35 \pm 0.94 \%$ respectively). All three values were significantly elevated in 48 (64\%) cases who had urine albuminuria measured qualitatively. There was positive co-relation of $\mathrm{HbAlC}$ with $\mathrm{Hs}$-CRP ( $r=0.713, p$ $=<0.0005)$, HbAlC with GGT ( $r=0.479, p=<0.00001)$ and Hs-CRP with GGT $(r=0.622, p=<0.0005)$. Conclusion: All three values were significantly positively correlated and elevated in diabetics, elevatedin those who had urine albuminuria (qualitative). This suggests possible role of inflammation, oxidant injury and glycemic control in the pathogenesis and progression of diabetic microvascular complications. GGT and Hs-CRP can be routinely used as early markers of diabetes microvascular complications.

Keywords: Diabetes Mellitus, $\mathrm{Hs}$-CRP, GGT, HbAlC, Inflammation, Oxidant injury, Albuminuria.
\end{abstract}

\section{Introduction}

Diabetes mellitus (DM) is a lifestyle disorder, the prevalence of which is increasing globally. Close to one-fifth of adults with diabetes mellitus in the world live in the South-East Asia region. International Diabetes Federation estimates that 382 million adults (8.3\%) - have diabetes mellitus, with people with diabetes progressing 
towards complications unaware ${ }^{[1]}$, thus resulting in associated morbidity and mortality. India is becoming the epicentre of diabetes mellitus in the world.

From chronic exposure to hyperglycemia results a state of chronic low-grade inflammation, which appears to be a major mechanism responsible for vascular (micro and macro) damage leading to complications of diabetes ${ }^{[2]}$, with the risk of complications increasing with degree of hyperglycemia.Chronic hyperglycemia is also postulated to cause oxidative stress via overproduction of superoxide radicals ${ }^{[2]}$, consuming NADPH and depleting glutathione, which leads to formation of advanced glycosylation end-products (AGE) of mitochondrial Electron Transport Chain (ETC) proteins resulting in their decreased function and further generation of free radicals.

High sensitivity C-reactive protein (Hs-CRP) is a C-reactive protein, an inflammatory marker, measured by high sensitivity assay to detect very low levels of CRP. Plasma levels of Hs-CRP provide a sensitive marker of increased low-grade inflammatory activity in arterial wall ${ }^{[3]}$. AGE induce and promote systemic vascular inflammation by releasing inflammatory mediators $^{[4]}$. This increases the levels of Hs-CRP. Gamma glutamyl transferase (GGT) is a cell surface protein maintaining intracellular antioxidant defense mechanism by contributing to extracellular catabolism glutathione $(\mathrm{GSH})^{[5]}$; with influx of its component amino acids to be utilized for intracellular glutathione synthesis. Hyperglycemia induced oxidative stress may increase serum GGT levels. Thus, GGT might reflect heperglycemia induced systemic oxidant injury.

One well known advanced glycosylation endproduct among diabetics is glycosylated hemoglobin $(\mathrm{HbA} 1 \mathrm{C})^{[6]}$. HbA1C reflects average glycemic control over previous 3 months. Because the development of complications is linked to accumulation of AGE in tissue proteins, measured by $\mathrm{HbA} 1 \mathrm{C}$, the core of the issue is glycemic control. Urine albuminuria can be used as an easy and qualitative assessment of diabetic microvascular complications.

There is a need to have alternate early markers for early steps in pathophysiology of diabetes, oxidative stress and chronic inflammation, to prevent progression and development of complications. Targeting inflammatory and oxidative pathways with use of early markers of inflammation and oxidant injury could possibly be a component of the strategies to prevent and control diabetes and related micro-vascular complications. There is a lack of sufficient data in Indian population regarding this, so is the need for this study.

\section{Material and Method}

This hospital based cross-sectional case-control study was conducted on 75 diabetic adult patients of $>40$ years age and 50 healthy age and sex matched controls in a tertiary care hospital in India. Each patient was subjected to detailed history and examination and recorded for comorbidities like coronary artery disease, cerebrovascular disease, peripheral vascular disease, chronic renal failure, malignancy, chronic liver disease or chronic respiratory disease.

Patients with Type 1 diabetes mellitus, alcohol consumption, hemolytic anemia or hemoglobinopathy, liver enzymes higher than three times the upper limit, on statins, NSAIDS, corticosteroids, methotrexate, amiodarone, tamoxifen or other hepatotoxic drugs, with obvious acute or chronic infection/vascular inflammation or any comorbid conditions were excluded from the study.

Diabetes was defined according to American Diabetes Association guidelines:

1. Diabetes symptoms (i.e. polyuria, polydipsia and unexplained weight loss) plus

a) Arandom venous plasma glucose concentration $\geq 11.1 \mathrm{mmol} / \mathrm{l}(\geq 200 \mathrm{mg} / \mathrm{dl})$ or 

b) A fasting
plasma
glucose
concentration $\geq 7.0$
$\mathrm{mmol} / \mathrm{l}$
(whole
blood $\geq 6.1 \mathrm{mmol} / \mathrm{l})(\geq 126 \mathrm{mg} / \mathrm{dl})$
or

c) Two-hour plasma glucose concentration $\geq 11.1 \mathrm{mmol} / 1(\geq 200 \mathrm{mg} / \mathrm{dl})$ two hours after $75 \mathrm{~g}$ anhydrous glucose in an oral glucose tolerance test (OGTT).

or

d) $\mathrm{HbA} 1 \mathrm{C} \geq 6.5 \%$

2. With no symptoms diagnosis to be based on atleast two glucose test on two different days with a value in the diabetic range either fasting, from a random sample or from the two-hour post glucose load.

Examination, routine investigations, quantitative assessment of Hs-CRP,HbA1C, GGT and qualitative estimation of urine albuminuria were done.HbA1C quantitative concentration was measured by dry chemistry kit; measured using QDxA1C, with readings compared to those obtained using high -pressure liquid chromatography. Serum GGT quantitative concentration was determined by end point essay using spectrophotometry. Quantitative determination of Hs-CRP in blood was done by ELISA.

Statistical Analysis: The data was entered in MS EXCEL spreadsheet and analysis was done using Statistical Package for Social Sciences (SPSS) version 21.0. Categorical variables were presented in number and percentage (\%) and continuous variables were presented as mean \pm Standard deviation (SD). Normality of data was tested by Kolmogorov-Smirnov test. If the normality was rejected, then non- parametric test was used. Quantitative variables were compared using unpaired t-test/Mann-Whitney Test (when the data sets were not normally distributed) between the two groups and ANOVA/Kruskal Wallis test (for non-parametric data) between more than two groups. Qualitative variables were compared using Chi-Square test /Fisher's exact test. Pearson correlation coefficient/Spearman correlation coefficient (for non-parametric data) was used to find out the association of GGT, Hs-CRP, HbA1C with each other. A $p$ value of $<0.05$ was considered statistically significant.

\section{Observation and Results}

The study was conducted in a tertiary care hospital in India on 75 adult cases (>40 years) of type 2 DM after fulfilling the inclusion and exclusion criteria and 50 age and sex matched healthy controls. Out of 75 cases, 38 were females and 37 were males. Control group included 25 females and 25 males. P-value was 0.942 , thus distribution of males and females in both groups was comparable. Mean age was $56.91 \pm 5.76$ years in diabetic cases and51.74 \pm 5.6 years in controls ( $\mathrm{p}$ $<0.0001$ ). Majority of cases (39) were in age group of 51-60 years whereas minimum number (13) were in age group of $<50$ years.

Interrelationship among Hs-CRP, HbA1C, GGT and urine albuminuria (qualitative) was studied. From our study following observations were made:

Mean value of Hs-CRP in DM cases was $8.94 \pm$ $2.54 \mathrm{mg} / \mathrm{Land} 1.52 \pm 0.98 \mathrm{mg} / \mathrm{L}$ in controls $(\mathrm{p}$ value $<0.0005$ ), with comparable Hs-CRP levels with gender distribution in cases $(9.07 \pm 2.78$ $\mathrm{mg} / \mathrm{L}$ in females, $8.81 \pm 2.23 \mathrm{mg} / \mathrm{L}$ in males, $\mathrm{p}$ value 0.657 ).

Mean value of GGT in DM cases was $101.61 \pm$ $36.84 \mathrm{U} / \mathrm{L}$ and $19.06 \pm 8.77 \mathrm{U} / \mathrm{L}$ in controls (pvalue $<0.0005$ ) with comparable GGT levels with gender distribution in cases $(99.26 \pm 38 \mathrm{U} / \mathrm{L}$ in females, $104.03 \pm 35.97 \mathrm{U} / \mathrm{L}$ in males, $\mathrm{p}$-value 0.579 ).

Mean value of $\mathrm{HbA} 1 \mathrm{C}$ was $9.24 \pm 1.97 \%$ in cases and $4.35 \pm 0.94$ in controls ( $\mathrm{p}$-value $<0.0005$ ) with comparable HbA1C levels with gender distribution in cases $(9.38 \pm 1.93 \%$ in females, $9.1 \pm 2.04 \%$ in males, p-value 0.433).

There was significant positive correlation of HbA1C with Hs-CRP ( $r=0.713, p=<0.0005)$ as shown in figure 1.

There was significant positive correlation of HbA1C with GGT $(r=0.479, p=<0.00001)$ as shown in figure 2. 
There was also significant positive correlation of Hs-CRP with GGT ( $r=0.622, p=<0.0005)$ as shown in figure 3.

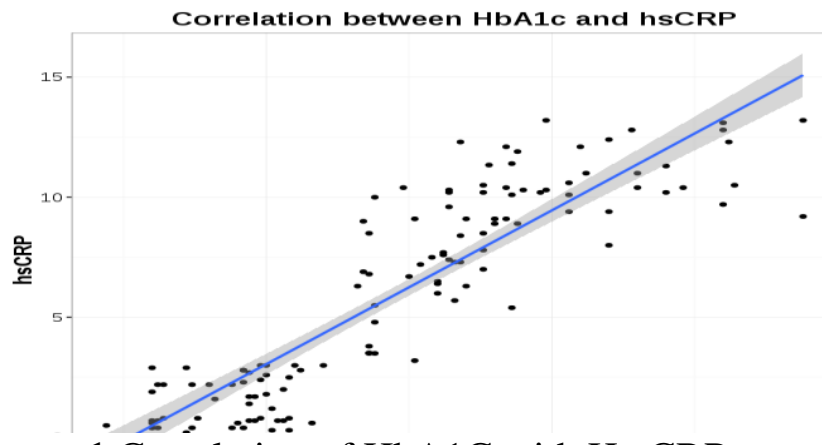

Figure 1 Correlation of HbA1C with Hs-CRP

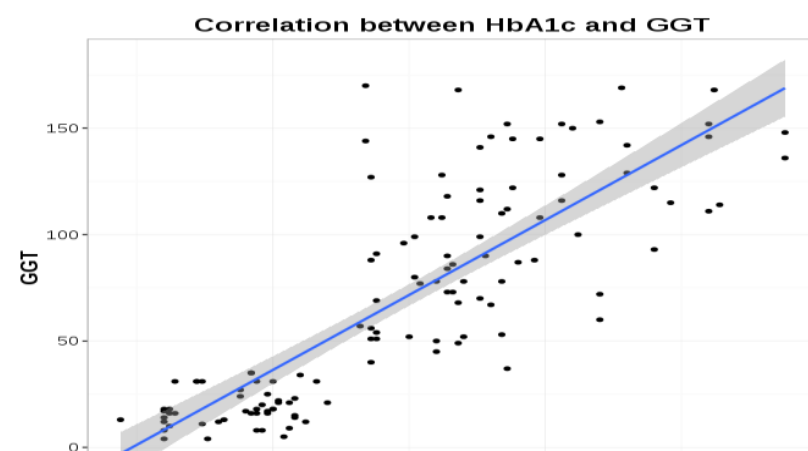

Figure 2 Correlation of $\mathrm{HbA1C}$ with GGT

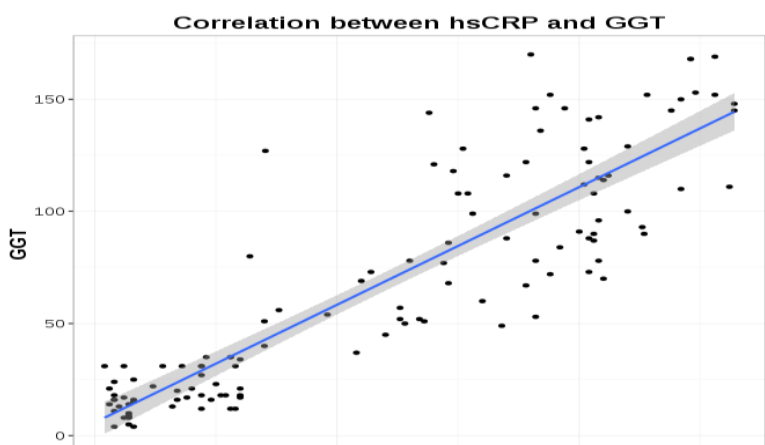

Figure 3 Correlation of Hs-CRP with GGT

As shown in table 1, controls had urine albumin findings of NIL. As mentioned in table 2, out of 75 type 2 DM cases, 27 (36\%) cases had NIL urine albumin, while $48(64 \%)$ cases had urine albuminuria of various grades measures qualitatively. Mean values of Hs-CRP, GGT and HbA1C were highest in cases with urine albumin $3+$.
Table 1 Urine albumin (qualitative) in controls

\begin{tabular}{|l|c|}
\hline Urine albumin & Controls $(\mathrm{N}=50)$ \\
\hline Nil & $50(100 \%)$ \\
\hline Trace & $0(0 \%)$ \\
\hline $1+$ & $0(0 \%)$ \\
\hline $2+$ & $0(0 \%)$ \\
\hline $3+$ & $0(0 \%)$ \\
\hline P-value & $<0.0005$ \\
\hline
\end{tabular}

Table 2 Urine albumin (qualitative) with GGT, $\mathrm{Hs}-\mathrm{CRP}$ and $\mathrm{HbA} 1 \mathrm{C}$ in cases

\begin{tabular}{|c|c|c|c|c|c|c|}
\hline $\begin{array}{c}\text { Urine } \\
\text { albumi } \\
\mathrm{n}\end{array}$ & Nil & Trace & $1+$ & $2+$ & $3+$ & $\begin{array}{c}\text { P- } \\
\text { value }\end{array}$ \\
\hline $\begin{array}{c}\text { Cases } \\
(\mathrm{N}=75\end{array}$ & $\begin{array}{c}27(36 \\
)\end{array}$ & $17(26.67 \%)$ & 14 & 13 & 4 & $<$ \\
GGT & $66.22 \pm$ & $107.18 \pm 26$. & $114.29 \pm 20$. & $140 \pm$ & $145 \pm$ & $<0.000$ \\
$(\mathrm{U} / \mathrm{L})$ & 22.14 & 74 & 63 & 20.05 & 24.21 & 1 \\
\hline $\mathrm{HbA} 1$ & $7.99 \pm$ & $8.64 \pm 1.7$ & $10.38 \pm$ & $10.29 \pm$ & 12.88 & $<$ \\
$\mathrm{C}(\%)$ & 1.19 & & 1.87 & 1.31 & \pm 1.4 & 0.0001 \\
\hline $\mathrm{Hs}-$ & $6.78 \pm$ & $8.79 \pm 1.83$ & $9.72 \pm 1.26$ & $11.64 \pm$ & 12.75 & $<$ \\
$\mathrm{CRP}$ & 1.99 & & & 1.04 & \pm 0.47 & 0.0001 \\
$(\mathrm{mg} / \mathrm{L}$ & & & & & & \\
\hline$)$ & & & & & & \\
\hline
\end{tabular}

\section{Discussion}

Diabetes mellitus (DM), with vascular damage leading to complications, is a state of chronic lowgrade inflammation and is postulated to cause oxidative stress via generation of free radicals ${ }^{[2]}$. Hyperglycemiain DM leads to formation of advanced glycosylation end-products (AGE) like HbA1C. AGE induce and promote systemic vascular inflammation, increasing the levels of Hs-CRP, a sensitive marker of low-grade inflammatory activity ${ }^{[3]}$. Hyperglycemia induced oxidative stress may increase serum GGT levels as GGT maintains intracellular antioxidant defense mechanism ${ }^{[5]}$.Urine albuminuria can be used as an easy and qualitative assessment of DM microvascular complications.

We studied the interrelationship among levels of Hs-CRP, HbA1C, GGT and urine albuminuria in75 type $2 \mathrm{DM}$ cases with $>40$ years ageand 50 age and sex matched healthy controls and thus assessing microvascular complications in them.

Mean value of $\mathrm{HbA1C}$ in DM cases was $9.24 \pm$ $1.97 \%$ and in controls was $4.35 \pm 0.94 \%$ (p-value $<0.0005)$ with comparable HbA1C levels with gender distribution ( $\mathrm{p}$-value of 0.433).

HbA1C and Hs-CRP: Mean value of Hs-CRP in DM cases was significantly higher than in controls (p-value < 0.0005), indicating ongoing 
inflammation in patients with type 2 DM. Correlation coefficient of HbA1C with Hs-CRP was statistically significant $(r=0.713$, p-value $<0.0005)$. As HbA1C levels increased in diabetic patients, Hs-CRP levels were also increased indicating more inflammatory response to poor glycemic control, considering Hs-CRP as an inflammatory marker.

Although there are very few studies of Hs-CRP levels in type $2 \mathrm{DM}$ patients from India, our results were in accordance with some of these studies. A cross-sectional study conducted by Gohel et $\mathrm{al}^{[7]}$ showed positive correlation of HbA1C with Hs-CRP values( $\mathrm{r}=0.78$, $\mathrm{p}$-value < $0.0001)$. Similarly, the study by Mahajan et $\mathrm{al}^{[8]}$ showed the median Hs-CRP levels were significantly higher in DM cases in both genders compared non-DM counterparts, independent of obesity (p-value<0.0001). Elevated Hs-CRP level was positively associated with type $2 \mathrm{DM}$ (OR 1.66; 95\% CI, p-value < 0.002). Another study by Meshram et al $^{[9]}$ also showed a significant increase of Hs-CRP in subjects with DM. Hs-CRP levels were significantly higher among the DM subjects $(5.1 \pm 2.9 \mathrm{mg} / \mathrm{L})$ than non-DM subjects $(1.01 \pm 0.2 \mathrm{mg} / \mathrm{L}), \mathrm{p}$-value $<0.05$. Similarly, the CURES-6 study ${ }^{[10]}$ also showed strong positive correlation of HbA1C with Hs-CRP, irrespective of age and gender (OR: 2.264, p-value <0.001), with Hs-CRP levels increased with increase in tertiles of HbA1C.

Our study findings were in accordance with some western studies. The study by Amanullah et al ${ }^{[11]}$ found that Hs-CRP was significantly higher among the DM subjects $(4.8 \mathrm{mg} / \mathrm{L})$ than non-DM subjects $(2.5 \mathrm{mgs} / \mathrm{L}), \mathrm{p}$-value $<0.001$. Hs-CRP showed a significant positive correlation with HbA1C ( $r=0.307$, p-value < 0.001).

HbA1C and GGT: Mean value of GGT in DM cases was significantly higher than in controls (pvalue $<0.0005$ ), indicating ongoing oxidant injury in patients of type $2 \mathrm{DM}$. Correlation coefficient ofHbA1C with GGT was statistically significant ( $\mathrm{r}$ $=0.479$, p-value <0.00001). As HbA1C levels increased in DM patients, GGT levels also increased, indicating more oxidant injury with poor glycemic control, considering GGT as an oxidant injury marker.

Very few studies have been done from India in this regard. Our findings were similar with those seen in the study by Gohel et $\mathrm{al}^{[7]}$ that showed positive correlation of $\mathrm{HbA1C}$ with GGT values $(r=0.79, p$-value $<0.0001)$.

Our study findings were in accordance with some western studies. A study by Sabanayagam et al ${ }^{[12]}$ showed that higher serum GGT levels were positively associated with type $2 \mathrm{DM}$, independent of alcohol consumption and other confounders. This positive association appeared to be present across the full range of GGT, without any threshold effect (OR: 2.33, p-value <0.0001). In the Mexico City Diabetes Study ${ }^{[13]}$, GGT was associated with prevalent type $2 \mathrm{DM}$ and as a predictor of type $2 \mathrm{DM}$ when followed up (OR: 14.7, p-value 0.0001).

Hs-CRP and GGT: Correlation coefficient of Hs-CRP with GGT was statistically significant ( $\mathrm{r}$ $=0.622$, p-value <0.0005). As Hs-CRP levels increased in DM patients, GGT levels also increased, linking inflammation with oxidant injury.

Very few studies have been done from India in this regard. Our findings were similar with those seen in the study by Gohel et $\mathrm{al}^{[7]}$ that showed positive correlation of Hs-CRP with GGT values $(\mathrm{r}=0.80$, $\mathrm{p}$-value $<0.0001)$, linking oxidant injury with inflammation.

Our study findings were in accordance with some western studies Also, in a cross-sectional study by Lee and Jacobs $\mathrm{Jr}^{[14]}$, serum level of GGT was closely associated with plasma CRP levels, serum concentration of GGT across all deciles was positively associated with serum concentrations of CRP (p-value for trend $<0.01$ ).

Hs-CRP, HBA1C and GGT: Very few studies were done with all these three variables together or showing any kind of correlation among the three variables. Our study findings were in accordance with the study by Gohel et $\mathrm{al}^{[7]}$ showed that serum GGT and Hs-CRP concentration were 
significantly increased in type $2 \mathrm{DM}$ more so with poor glycemic control. There was a significant positive correlation between serum GGT activity and Hs-CRP. Both were independently and positively correlated with $\mathrm{HbA} 1 \mathrm{C}$.

Urine Albuminuria: Urine albuminuria was used as an easy and qualitative assessment of diabetic microvascular complications. With reference to table 2, out of 75 type 2 DM cases, 27 (36\%) cases had NIL urine albumin, while 48 (64\%) cases had urine albuminuria of various grades measures qualitatively. Mean values of Hs-CRP, GGT and HbA1C were significantly elevated with qualitative urine albuminuria (p-value $<0.0001$ ), with values of Hs-CRP, HbA1C and GGT highest in cases with highest grade albuminuria (3+) and lowest with urine albumin NIL. These findings implied progress in diabetic micro-vascular complications with increasing levels of inflammation, oxidant injury and poor glycemic control.

Our findings were in accordance with study by Holman et $\mathrm{al}^{[15]}$ which showed that increase in HbA1C had a persistent effect on complications several years after their measurement, the risk of micro and macrovascular complications increased with the increase in HbA1C levels.

The disparity with our study could be due to cross-sectional nature of study hence incidence of diabetes and effects on values of GGT and HsCRP by drugs for diabetes could not be calculated. Albuminuria was assessed qualitatively. Standardized Hs-CRP and GGT baseline values were not available for Indian population. Large studies are required to consolidate the above findings which may lead to new therapeutic strategies.

Thus, we conclude that Hs-CRP,HbA1C, GGT have been found to be correlated significantly with each other, with higher levels with poor glycemic control and higher values with higher grade urine albuminuria, suggesting the possible role of inflammation, oxidant injury and glycemic control in the progression of microvascular complications of diabetes mellitus.GGT and Hs-
CRP can be used routinely as early markers for inflammation and oxidant injury respectively and for development of complications as well to prognosticate microvascular complications associated with poor glycemic control.

\section{References}

1. Farsani SF, Van-der-Aa MP, Van-derVorst MMJ, Knibbe CAJ, de Boer A. Global trends in the incidence and prevalence of type 2 diabetes in children and adolescents: a systematic review and evaluation of methodological approaches. Diabetologia.2013;56:1471-1488.

2. Brownlee M. Biochemistry and molecular cell biology of diabetic complications. Nature. 2001; 414:813-820.

3. Clyne B, Olshaker J. The C-reactive protein. J Emerg Med.1999;17(6):10191025.

4. Giugliano D, Marfella R, Coppola L. Vascular effects of acute hyperglycemia in humans reversed by 1-Arginine. Evidence for reduced availability of nitric oxide duringhyperglycemia.Circulation.1997;95( 7):1783-1790.

5. Karp DR, Shimooku K, Lipsky PE. Expression of gamma-glutamyl transpeptidase protects ramos B cells from oxidation induced cell death. J Biol Chem.2001;276:3798-3804.

6. Nathan DM, Balkau B, Bonara E. International expert committee report on the role of $\mathrm{A} 1 \mathrm{C}$ assay in diagnosis of diabetes. Diabetes Care.2009;32(7):13271334.

7. Gohel MG, Chakho AN. Serum GGT activity and hsCRP level in patients with type 2 diabetes mellitus with good and poor glycemic control: An evidence linking oxidative stress, inflammation and glycemic control. Journal of Diabetes and Metabolic Disorders.2013;12(56):1-8.

8. Mahajan A, Tabassum R, Chaval S, Dwivedi OP, Bharadwaj M, Tandon N, 
Bharadwaj D. J Clin Endocrin Metab. 2009;94(6):2123-2127.

9. Meshram A, Agrawal U, Dhok A, Adole P, Meshram K, Khare R. HbA1c, hs-CRP and anthropometric parameters evaluation in the patients of Diabetes Mellitus of Central Rural India. Int J Med Sci Public Health.2013;2(2):293-296.

10. Mohan V, Deepa R, Velmurugan K, Premalatha G. Association of C-Reactive Protein with body fat, diabetes and coronary artery disease in Asian Indians: The Chennai Urban Rural Epidemiology Study (CURES-6). J Clin Endocrin Metab.2009;94(6):2123-2127.

11. Amanullah S, Jarari A, Govindan M, Basha MI, Khatheeja S. Association of hsCRP with Diabetic and Non-diabetic individuals. Jordan Journal of Biological Sciences.2010;3:7-12.

12. Sabanayagm C, Shankar A, Li J, Pollard C, Ducatman A. Serum gamma-glutamyl transferase level and diabetes among US adults. EUR J of Epidimiol. 2009; 24(7):369-373.

13. Nannipieri M, Gonzales C, Baldi S. Liver enzymes, the metabolic syndrome, and incident diabetes: the Mexico City diabetes study. Diabetes Care.2005;28(7):17571762.

14. Lee DH, Jacobs DR Jr. Association between serum Gamma-glutamyl transferase and C-reactiveprotein. Atherosclerosis. 2005;178(2):327-330.

15. Holman R, Paul S, Bethel A, Matthews D, Neil A. 10-Year Follow-up of Intensive Glucose Control in Type 2 Diabetes. N Engl J Med.2008;359(15):1577-1589. 